\title{
Monitoreo de la implementación del programa Chile Crece Contigo en el embarazo: 2008 - 2011
}

\author{
Eduardo Atalah S. ${ }^{1}$, Miguel Cordero V. ${ }^{2}$, Susana Quezada L. ${ }^{3}$, Ximena Carrasco F. ${ }^{3}$, \\ Marcela Romo M.4, María Elizabeth Guerra Z.5 \\ ${ }^{1}$ Departamento de Nutrición, Facultad de Medicina, Universidad de Chile. ${ }^{2}$ School of Social and Community Medicine, \\ University of Bristol, UK. ${ }^{3}$ Departamento de Estadísticas e Información de Salud, Ministerio de Salud. ${ }^{4}$ Escuela de \\ Nutrición y Dietética, Facultad de Medicina, Universidad de Chile. ${ }^{5}$ Becaria CONICYT, Programa Doctorado en Salud \\ Pública, Universidad de Chile.
}

Estudio financiado por el proyecto 106817-001 /002, IDRC, Canadá.

\section{RESUMEN}

Objetivo: Evaluar el grado de implementación de las actividades de Chile Crece Contigo en el embarazo y parto en los 29 Servicios de Salud del país. Método: Análisis de bases de datos del DEIS, Ministerio de Salud 2008 y 2011 por Servicios de Salud. Se analizó mediana y percentiles de gestantes en riesgo psicosocial, visitas domiciliarias integrales, participación de madres en talleres educativos, parto acompañado y apego precoz y el cambio de cada uno de ellos en el período estudiado. En función de la magnitud de los cambios se generó un nuevo indicador que permitió clasificar a los Servicios de Salud en terciles, considerando el tercil superior aquellos con cambios más positivos. Resultados: Ingresaron a control prenatal cerca de 200 mil mujeres cada año, que representan aproximadamente el $80 \%$ de los recién nacidos del país. Se observó un aumento significativo de 16 a 22 puntos porcentuales en todas las variables estudiadas $(p<0,005)$, excepto en visitas domiciliarias en gestantes en riesgo psicosocial, que disminuyeron de 1,6 a $1,1(p<0,001)$, con variaciones importantes entre Servicios de Salud. Conclusión: Ha habido un esfuerzo importante para modernizar el Programa de la Mujer y mejorar el control prenatal, parto y puerperio, aunque no se ha reflejado en los indicadores de salud. Las diferencias de comportamiento por Servicios de Salud ofrece la oportunidad de profundizar en el análisis de las causas que lo explican y la posibilidad de definir estrategias para lograr mejores resultados

\section{PALABRAS CLAVES: Embarazo, Chile Crece Contigo, monitoreo, control prenatal, políticas públicas}

\section{SUMMARY}

Objective: To assess the degree of implementation of the activities of Chile Crece Contigo in pregnancy and childbirth in the 29 country's health services. Method: Analysis of DEIS databases, Ministry of Health 2008 and 2011 by Health Services. Median and percentiles were analyzed at psychosocial risk in pregnant women, comprehensive home visits, mothers participate in educational workshops, childbirth accompanied and early bonding and changing during the study period. Depending on the magnitude of the changes generated a new indicator that allowed classification of Health Services in tertiles, the highest tertile considering those with more positive changes. Results: prenatal entered nearly 200,000 women each year, approximately $80 \%$ of all pregnant women in the country. We observed a significant increase of 16-22 percentage points for all variables studied $(p<0.005)$, except home visits to pregnant women in psychosocial risk decreased from 1.6 to $1.1(p<0.001)$, with important variations by Health Services. Conclusion: There has been a major effort 
to modernize the Program for Women and improving prenatal care, childbirth and postpartum, although not reflected in health indicators. The differences in behavior Health Services offers the opportunity to deepen the analysis of the causes that explain and the ability to define strategies to achieve better results.

\section{KEYS WORDS: Pregnancy, Chile Crece Contigo, monitoring, prenatal care, public policies}

\section{INTRODUCCIÓN}

Chile ha avanzado en forma importante en políticas públicas en relación a la madre y el niño, logrando muy buenos indicadores de morbimortalidad en esta población (1-3). Sin embargo, el desarrollo integral de los niños y la calidad de vida en la infancia muestran cifras preocupantes. La Encuesta Nacional de Calidad de Vida (ENCAVI 2006) mostró que el $29,9 \%$ de los menores de 5 años presentaba algún grado de riesgo o retraso en el nivel de desarrollo esperable para su edad (4). Al igual que la mayor parte de los indicadores de salud, existen importantes inequidades en la prevalencia de déficit del desarrollo en función del quintil de ingreso familiar (4). Por otra parte existe una baja cobertura de educación preescolar, especialmente en los grupos más vulnerables y por lo tanto el contacto principal entre el Estado y esta población se da desde el ámbito sanitario (5).

En este escenario se genera el Sistema Intersectorial de Protección Social con acciones y prestaciones ejecutadas por distintos organismos del Estado, destinadas a la población más vulnerable. Uno de los subsistemas que lo componen es Chile Crece Contigo ( $\mathrm{CHCC}$ ), que consiste en entregar acompañamiento y seguimiento personalizado a la trayectoria del desarrollo de los niños y niñas, incluyendo principios orientadores y prestaciones garantizadas desde la gestación $(6,7)$.

El eje de CHCC es el Programa de Apoyo al Desarrollo Biopsicosocial (PADB), ejecutado por el componente de salud en sus distintos niveles de atención, siendo el hito de entrada al sistema el primer control de salud prenatal y el hito de salida cuando el niño entra al sistema formal de educación, vale decir, el primer nivel de transición de educación parvularia $(8,9)$.

La instalación de CHCC se realizó en dos etapas: 161 de las comunas del país ingresaron el 2007 y las 181 restantes durante el 2008. La instalación se planteó desde su diseño como progresiva, seleccionándose aquellas comunas en que existieran mejores condiciones para la implementación.

Recientes estudios sobre el impacto de $\mathrm{CHCC}$, permiten concluir que se logra mejor nivel de desarrollo infantil cuando las prestaciones se incorporan a más temprana edad de los niños, cuando la calidad de la implementación es más adecuada y que se obtiene mayor impacto en las madres más vulnerables (10-12)
Aún cuando el objetivo final está centrado en el niño, existen diversas actividades programas durante la gestación. El objetivo de este estudio fue monitorear la implementación de las actividades planificadas en el embarazo y parto en cada una de los 29 Servicios de Salud del país, comparando la cobertura de estas actividades entre el 2008 y 2011. En una segunda etapa se hará un análisis similar en relación a las actividades programadas en el niño, para finalmente concluir con un estudio cualitativo que permita identificar los factores que pueden contribuir a lograr mejores resultados en el programa.

\section{MATERIAL Y MÉTODO}

La información se obtuvo de las bases de datos del Departamento de Estadísticas e Información en Salud (DEIS) del Ministerio de Salud. Estas se generan a partir de los Resúmenes Estadísticos Mensuales (REM), que representan la fuente oficial para la captación de los datos básicos requeridos para la gestión central y local de todos los establecimientos de la Red Pública Asistencial del país.

En función de la información disponible se generaron 5 indicadores que se describen a continuación, considerando como población bajo control la población de gestantes a diciembre de 2008 y 2011. Se decidió analizar los cambios observados en un período de 3 años, considerando la complejidad de implementar a nivel nacional un proyecto de esta envergadura.

Gestantes en riesgo psicosocial: Porcentaje de madres en riesgo psicosocial en relación al total se madres en que se aplicó la encuesta. En el primer control prenatal se aplica una pauta estandarizada para la detección temprana del riesgo psicosocial (Evaluación Psicosocial Abreviada, EPsA). Este instrumento permite pesquisar factores de riesgo que pueden afectar el bienestar de la madre y el desarrollo integral del niño(a) antes de nacer. Para una adecuada aplicación de la EPsA se aumentó el tiempo de duración del primer control de gestación de 20 a 40 minutos, lo que sin duda contribuyó a una mayor sensación de apoyo y a detectar activamente las familias que requieren apoyos especiales. Los resultados de esta pauta permiten priorizar acciones en salud y activar prestaciones diferenciadas de la Red de Protección Social según corresponda. 
Tasa de visitas domiciliarias integrales: Número de visitas realizadas en relación al total de madres en riesgo psicosocial. Son realizadas por integrantes del equipo de salud y su principal objetivo es la promoción de mejores condiciones ambientales y de relación familiar, para favorecer la salud materna y el desarrollo integral en la primera infancia.

Participación de madres en talleres educativos: Número de madres que participan en talleres en relación al total de embarazadas en control prenatal. Corresponden a talleres de educación grupal de preparación para el parto y la crianza, dirigidos a las mujeres y sus parejas o acompañantes. Tienen la finalidad de dar apoyo cognitivo y emocional durante el embarazo, aumentar la capacidad de autocuidado y bienestar emocional y fortalecer la preparación física y emocional para el parto y la crianza en el primer período del recién nacido(a). Los talleres constan de 6 sesiones (4 en atención primaria y dos en la maternidad), en las cuales se pretende favorecer la formación del vínculo seguro, aprender las distintas posiciones que se pueden adoptar durante el trabajo de parto y parto, promover el vínculo madre, padre e hijo(a), fortalecer el rol del padre en el proceso de gestación, nacimiento y crianza y promover la lactancia materna.

Parto acompañado: Porcentaje de partos con participación del padre o familiar cercano en relación al total de partos. Un foco primordial de las acciones educativas de Chile Crece Contigo es promover la participación activa de los hombres durante todo el proceso de la gestación, parto y crianza de sus hijos(as). La percepción de apoyo de la pareja se asocia a bienestar emocional de la madre y además el cuidado directo del recién nacido(a) por el padre, promueve el establecimiento de interacciones sensibles que llevan al establecimiento de un vínculo seguro entre ellos.

Apego precoz: Porcentaje de recién nacidos con contacto piel a piel, sobre el vientre y pecho de su madre y amamantamiento los primeros 20-30 minutos post parto en relación al total de partos. Esta actividad contribuye al fomento de la lactancia materna y el vínculo madre-hijo a través de campañas publicitarias y material educativo para los equipos de salud.

Análisis estadístico. Se determinaron estos cinco indicadores en cada uno de los 29 Servicios de Salud del Sistema Público de Salud para el año 2008 y 2011. Se analizó la normalidad de cada variable y dado que ninguno de los indicadores presentó un comportamiento normal, se utilizó la mediana y percentiles como variable de tendencia central. Para evaluar los cambios entre ambos períodos se aplicó el Sign Rank Test, considerándose como significativo un valor de $p<0,05$.

Adicionalmente se determinó la magnitud del cambio de cada indicador entre ambos períodos para cada Servicio de Salud, expresados como puntos porcentuales. Este valor fue posteriormente categorizado en terciles, asignando un valor 1 para el grupo con menor cambio (primer tercil) y 3 para el tercil superior. Se excluyó de este análisis la proporción de gestantes en las cuales se aplicó la pauta de Evaluación Psicosocial Abreviada, ya que ello no representa una intervención propiamente tal del programa. La suma de este valor para las cuatro variables estudiadas permitió generar un nuevo indicador, el que también fue categorizado por terciles para identificar los Servicios de Salud con mejores resultados en la implementación del programa en este período.

\section{RESULTADOS}

En el período estudiado ingresaron anualmente a control prenatal cerca de 200 mil mujeres en el sistema público de salud, que representan aproximadamente el $80 \%$ del total de nacidos vivos del país. El total de actividades realizadas en este período, en números absolutos, se presenta en la Tabla I, destacando un aumento importante en todas ellas. Es necesario tener presente que existen diferencias importantes en relación al tamaño de la población atendida por Servicio de Salud, con una mediana de 5.704 embarazadas (p25-75: 4.3869.115) y valores extremos de 1.405 y 17.627 .

A la mayor parte de las gestantes que ingresaron a control se les aplicó la pauta de evaluación

\section{Tabla I \\ POBLACIÓN ATENDIDA Y ACTIVIDADES REALIZADAS EN EL SISTEMA PÚBLICO DE} SALUD: 2008 Y 2011

\begin{tabular}{lcc}
\hline Variable & 2008 & 2011 \\
\hline $\begin{array}{l}\text { Gestantes ingresadas a } \\
\text { control prenatal }\end{array}$ & 202.755 & 197.064 \\
$\begin{array}{l}\text { Gestantes en riesgo } \\
\text { psicosocial }\end{array}$ & 30.995 & 75.117 \\
$\begin{array}{l}\text { Visitas domiciliarias } \\
\text { integradas }\end{array}$ & 49.100 & 85.250 \\
$\begin{array}{l}\text { Ingresos a Talleres de } \\
\text { Educación }\end{array}$ & 138.296 & 175.817 \\
$\begin{array}{l}\text { Total de partos } \\
\text { Partos con participación } \\
\text { del padre }\end{array}$ & 118.999 & 132.299 \\
$\begin{array}{l}\text { Partos con apego } \\
\text { precoz }\end{array}$ & $54.789^{\star}$ & 101.178 \\
\hline
\end{tabular}

*Información del 2009. 
del riesgo psicosocial, detectándose que una de cada cuatro de las gestantes estaban en riesgo psicosocial el 2008, proporción que aumentó significativamente el 2011 (Tabla II) lo que podría explicarse a un aumento en la pesquisa y a la instalación progresiva del modelo CHCC. Destaca una amplia variabilidad entre los servicios de salud, reflejada en la distribución percentilar.

En la línea base (2008) cada madre en riesgo psicosocial recibió una mediana de 1,6 visitas domiciliarias, proporción que disminuyó significativamente al término de esta evaluación, reflejando una debilidad del programa en esta variable. Este hecho en parte se explicaría por el aumento en la proporción de madres en riesgo, ya que el número absoluto de visitas aumentó en $70 \%$. En los otros tres indicadores se observó un comportamiento positivo, con un incremento de cerca de 20 puntos porcentuales entre el 2008 y 2011 , destacando también la variabilidad entre los Servicios de Salud.

La mediana de cambios entre el 2008 y el 2011 por Servicios de Salud y su distribución percentilar se muestra en la Tabla III. Los resultados refuerzan los datos anteriores, mostrando cambios positivos en cada indicador, a excepción de las visitas domiciliarias, que aumentaron en número absoluto, pero que disminuyeron al considerar como denominador las familias en riesgo psicosocial. Nuevamente se observan variaciones importantes entre Servicios de Salud al considerar los percentiles 25 y 75 .

El puntaje total obtenido en los 4 indicadores evaluados según terciles se muestra en la Tabla IV. Seis Servicios obtuvieron menos de 7 puntos, estando en la mayoría de las variables en el tercil inferior. Existen algunas excepciones, como Aisén por ejemplo incluido en el tercil inferior, que está en la categoría superior en cuanto a partos acompañados por el padre, lo que en parte se puede explicar por una menor población beneficiaria, que puede determinar mayor fluctuación en las cifras. En el otro extremo tres Servicios obtuvieron 11 puntos y más, estando en el tercil superior prácticamente en las cuatro variables estudiadas.

\section{DISCUSIÓN}

A pesar de la importancia y amplia cobertura de Chile Crece Contigo, existe una limitada evaluación del proceso de implementación y del eventual impacto del programa. Actualmente se desarrolla una iniciativa para evaluar el impacto en el desarrollo psicomotor de los niños, información que aún no está disponible (13).

Como una etapa previa es interesante conocer el grado de cumplimiento de las distintas actividades programadas, tanto en la madre como en el niño. Este es el propósito del estudio, que centró el análisis en los indicadores del embarazo y parto, para después complementarlo con las actividades programadas en el niño.

La información del estudio se basa en los datos del Departamento de Estadísticas e Información en

Tabla III

MEDIANA DE CAMBIO EN LOS INDICADORES DE LA GESTANTE ENTRE EL 2008 Y 2011 (PUNTOS PORCENTUALES)

\begin{tabular}{lcc}
\hline Indicador & Mediana & Percentil 25-75 \\
\hline $\begin{array}{l}\text { Gestantes en riesgo } \\
\text { psicosocial }\end{array}$ & 18,1 & $13,1-26,3$ \\
$\begin{array}{l}\text { Visitas domiciliarias } \\
\text { Integrales/familias en } \\
\text { riesgo }\end{array}$ & $-0,4$ & $-1,5--0,1$ \\
$\begin{array}{l}\text { Ingreso talleres de } \\
\text { educación de grupo }\end{array}$ & 12,8 & $-0,8-43,3$ \\
$\begin{array}{l}\text { Partos con participa- } \\
\text { ción del padre }\end{array}$ & 13,1 & $1,9-23,4$ \\
$\begin{array}{l}\text { Partos con apego } \\
\text { precoz }\end{array}$ & 30,0 & $6,7-44,5$ \\
\hline
\end{tabular}

Tabla II

MEDIANA DE CAMBIO EN LOS INDICADORES DE LA GESTANTE ENTRE 2008 Y 2011

\begin{tabular}{lccccc}
\hline Indicador & \multicolumn{2}{c}{2008} & \multicolumn{2}{c}{2011} & \multicolumn{2}{c}{ Valor p } \\
\hline Gestantes en riesgo psicosocial & $p 50$ & $p$ 25-75 & $p$ 50 & p 25-75 & $<0,001$ \\
Visitas domiciliarias integrales & 25,0 & $16,3-33,9$ & 42,0 & $35,7-54,0$ & $<0,001$ \\
Ingreso talleres educación grupal & 1,6 & $1,1-2,8$ & 1,1 & $0,9-1,4$ & $<0,005$ \\
Partos con participación paterna & 73,0 & $45,9-96,3$ & 95,1 & $74,3-107,9$ & $<0,001$ \\
Partos con apego precoz & 67,7 & $58,6-77,5$ & 83,8 & $77,0-88,1$ & $<0,002$ \\
\hline
\end{tabular}


Tabla IV

DISTRIBUCION EN TERCILES DEL PUNTAJE DE CADA SERVICIO DE SALUD CONSIDERANDO LA MAGNITUD DEL CAMBIO ENTRE 2008 Y 2011

\begin{tabular}{|c|c|c|c|c|c|}
\hline Servicio de Salud & $\begin{array}{c}\text { Visitas } \\
\text { domiciliarias }\end{array}$ & $\begin{array}{c}\text { Talleres } \\
\text { gestantes }\end{array}$ & $\begin{array}{c}\text { Parto } \\
\text { acompañado }\end{array}$ & $\begin{array}{l}\text { Apego } \\
\text { precoz }\end{array}$ & $\begin{array}{c}\text { Puntos } \\
\text { Total }\end{array}$ \\
\hline \multicolumn{6}{|l|}{ Tercil inferior } \\
\hline Osorno & 2 & 1 & 1 & 1 & 5 \\
\hline Valparaíso-San Antonio & 1 & 1 & 1 & 2 & 5 \\
\hline Aisén & 1 & 1 & 3 & 1 & 6 \\
\hline Atacama & 1 & 2 & 2 & 1 & 6 \\
\hline Coquimbo & 2 & 2 & 1 & 1 & 6 \\
\hline Magallanes & 1 & 2 & 2 & 1 & 6 \\
\hline Araucanía Sur & 1 & 2 & 2 & 2 & 7 \\
\hline Concepción & 1 & 2 & 2 & 2 & 7 \\
\hline Iquique & 1 & 1 & 2 & 3 & 7 \\
\hline Metropolitana Norte & 2 & 1 & 1 & 3 & 7 \\
\hline Metroplitana Sur-Oriente & 2 & 2 & 1 & 2 & 7 \\
\hline Ñuble & 2 & 3 & 1 & 1 & 7 \\
\hline \multicolumn{6}{|l|}{ Tercil medio } \\
\hline Chiloé & 2 & 1 & 3 & 2 & 8 \\
\hline Del Libertador & 1 & 2 & 2 & 3 & 8 \\
\hline Metropolitana Sur & 3 & 1 & 1 & 3 & 8 \\
\hline Viña-Quillota & 1 & 3 & 2 & 2 & 8 \\
\hline Araucanía norte & 2 & 3 & 3 & 1 & 9 \\
\hline Bío-Bío & 2 & 2 & 3 & 2 & 9 \\
\hline Del Maule & 3 & 3 & 1 & 2 & 9 \\
\hline Metropolitana Central & 3 & 3 & 1 & 2 & 9 \\
\hline Metropolitana Occidente & 3 & 3 & 2 & 1 & 9 \\
\hline Valdivia & 2 & 1 & 3 & 3 & 9 \\
\hline \multicolumn{6}{|l|}{ Tercil Superior } \\
\hline Aconcagua & 3 & 1 & 3 & 3 & 10 \\
\hline Arauco & 3 & 3 & 2 & 2 & 10 \\
\hline Arica & 3 & 3 & 3 & 1 & 10 \\
\hline Metropolitana Oriente & 3 & 2 & 2 & 3 & 10 \\
\hline Antofagasta & 2 & 3 & 3 & 3 & 11 \\
\hline Talcahuano & 3 & 2 & 3 & 3 & 11 \\
\hline Del Reloncaví & 3 & 3 & 3 & 3 & 12 \\
\hline
\end{tabular}

1= Tercil inferior; 2 = Tercil medio; 3 = Tercil superior

Salud (DEIS) del Ministerio de Salud, que tienen la virtud de representar al universo de establecimientos de la Red Pública Asistencial del país, de generar indicadores bien estandarizados y en forma periódica. Sin embargo, puede haber un cierto grado de duda sobre la calidad y confiabilidad de la información. Así por ejemplo en este análisis el ingreso a Talleres Educativos muestra un valor superior al
$100 \%$ en un Servicio de Salud, lo que debe representar un error. Si la misma evaluación se realiza a nivel de Centros de Salud, está situación es más frecuente. La calidad de la información se relaciona directamente con la supervisión de los referentes técnicos que solicitan los datos obtenidos en el registro estadístico mensual (REM). Año a año el DEIS ha aumentado el volumen de datos a validar 
por la mayor demanda de información, superando actualmente 12.000 .000 de datos por año, lo que hace muy compleja esta tarea.

A pesar de esta debilidad es interesante evaluar el grado de cobertura de las actividades programadas por Servicios de Salud, lo que permite identificar puntos críticos donde poner mayor énfasis en la implementación. Una duda metodológica inicial fue decidir si el nivel de cambio de cada indicador se evaluaba como porcentaje de cambio del valor inicial, o simplemente como puntos porcentuales. Optamos por la segunda opción, considerando que si un Servicio tenía un valor inicial muy bajo podía incrementar su cobertura en $300-400 \%$, superando con creces a aquellos Servicios que aumentaron más su cobertura en términos absolutos.

En los resultados llama la atención un importante aumento en las gestantes en riesgo psicosocial, lo que es difícil de explicar por variaciones negativas en las condiciones de vida en este período. Este hecho podría ser el resultado más bien de una mayor capacitación o mayor sensibilidad del equipo de salud para detectar los problemas de riesgo psicosocial. El mayor diagnóstico de riesgo psicosocial determinó un impacto negativo en la cobertura de visitas domiciliarias, las que aumentaron en número absoluto, pero disminuyeron al considerar como denominador las familias en riesgo psicosocial.

De este análisis se desprende además que hay grandes diferencias en los resultados entre los 29 servicios de salud del país, y probablemente aún más entre las 356 comunas, aunque ello no fue evaluado en esta ocasión. Así por ejemplo destacan los Servicios de Salud de Antofagasta, Talcahuano y Concepción, que se ubicaron en el tercil superior en al menos 3 de los 4 indicadores analizados. Ello abre una ventana de oportunidades para identificar las mejoras prácticas utilizadas en los distintos niveles y sacar lecciones que puedan ser compartidas con los diferentes participantes del sistema, organizaciones públicas y grupos académicos.

Aún cuando el programa no ha definido indicadores de impacto en relación a la embarazada, parto, peso al nacer y lactancia materna, no se han observado cambios positivos en ningunos de estos indicadores. La prevalencia de obesidad de la embarazada sigue aumentando, existe una alta prevalencia de cesáreas (mediana: 35,6; p25-75: 30,9-45,4), de macrosomía fetal $(10,3 \%)$ y ha descendido la lactancia materna exclusiva a los 6 meses $(14,15)$. Este último hecho podría estar vinculado con la introducción en el año 2008 de la bebida láctea Purita Mamá en el Programa Nacional de Alimentación Complementaria (PNAC), que tiene menor aceptabilidad en relación producto entregado anteriormente la Leche Purita Fortificada. Dado que las mujeres que no están con lactancia exclusiva reciben Leche Purita Fortificada, aparentemente las usuarias estarían informando la suspensión de la lactancia, sin que esto sea efectivo en todos los casos, para recibir el producto de mayor aceptabilidad $(16,17)$. Ello podría explicar la aparente disminución de lactancia materna exclusiva a pesar de todas las acciones educativas del Chile Crece Contigo y el fomento del apego pre$\mathrm{coz}$, siendo necesario otros estudios para verificar la real magnitud de la lactancia materna exclusiva al sexto mes.

\section{CONCLUSIÓN}

Ha habido un esfuerzo importante para modernizar el Programa de la Mujer y mejorar el control prenatal, parto y puerperio, pero que ello aún no se ha reflejado en los indicadores de salud. Considerando la magnitud y complejidad del programa, probablemente se requiere un plazo mayor para una adecuada implementación y lograr impactos en salud. Las diferencias de comportamiento por Servicios de Salud ofrece la oportunidad de profundizar en el análisis de las causas que lo explican y la posibilidad de definir estrategias para lograr mejores resultados.

\section{REFERENCIAS}

1. World Health Organization. World Health Statistics 2013, Geneve 2013. Disponible en: http://www.who. int/gho/publications/world_health_statistics/2013/en/ index.html

2. González R. Salud Materno-Infantil en las Américas. Rev Chil Obstet Ginecol 2010;75:411-21.

3. González R. Chile: nuevos desafíos en políticas públicas materno-infantiles. Rev Chil Obstet Ginecol 2012;77:329-30.

4. Ministerio de Salud. II Encuesta Nacional de Calidad de Vida y Salud 2006. Santiago 2007. Disponible en: http:// epi.minsal.cl/epi/html/sdesalud/calidaddevida2006/lnforme $\% 20$ Final\%20Encuesta\%20de\%20Calidad $\% 20$ de\%20Vida\%20y\%20Salud\%202006.pdf

5. Arzola MP, Camhi R. Educación Preescolar: Evidencia y desafíos para Chile. Libertad y Desarrollo, Serie de Informe Social. Santiago, 2013. Disponible en: http:// www.lyd.com/temaspublicos/editora/SISO138Educpreescolar\%20\%281\%29.pdf

6. El futuro de los niños es siempre hoy. Propuestas del Consejo Asesor Presidencial para la reforma de las políticas de infancia. Junio 2006. Disponible en: http:// www.oei.es/inicial/futuro_ninos_siempre_hoy_chile.pdf

7. Villar E. Los determinantes sociales de salud y la lucha por la equidad en salud: desafíos para el estado y la sociedad civil. Saúde Soc 2007;16:7-13.

8. MINSAL. Manual para el apoyo y seguimiento de desarrollo psicosocial de los niños y niñas de 0 a 6 años. Ministerio de Salud, Chile 2008. Disponible en: http:// es.scribd.com/doc/137606797/Manual-Para-El-Apoyo-y-Seguimiento-Del-Desarrollo-Psicosocial-de-LosNinos-y-Ninas-de-0-a-6-Anos-2008

9. Cuatro años creciendo juntos. Memoria de la instalación del Sistema de Protección Integral a la Infancia, 
Chile Crece Contigo 2006-2010. Silva V, Molina H (Eds). Santiago, 2010. Disponible en: http://www.fundacionpobreza.cl/biblioteca-archivos/memoria-chilecrece-contigo.pdf

10. Asesorías para el desarrollo. Evaluación de Impacto del Sistema de Protección Integral a la Infancia (Chile Crece Contigo). Santiago, 2011.

11. López M. Chile Crece Contigo: Lecciones para el diseño e implementación de políticas sobre protección a la primera infancia. Tesis para optar al grado de Magister Gestión y Políticas Públicas Universidad de Chile, 2009.

12. Ochoa G, Maillard C, Solar X. Primera infancia y políticas públicas, una aproximación al caso del Sistema Integral de Protección a la Infancia Chile Crece Contigo. Santiago de Chile, 2010. Disponible en: http:// www.germina.cl/wp-content/uploads/2011/05/publicacion4_infancia_politicas_publicas_germina2010.pdf

13. Encuesta Longitudinal de la Primera Infancia. Ministerio de Salud, Centro Microdatos, Facultad de Econo- mía, Universidad de Chile. Disponible en: http://www. elpi.cl/

14. Ministerio de Salud. Tendencia en la situación nutricional de la población chilena controlada en el sistema público de salud 2008-2010, Santiago 2011. Disponible en: http://www.minsal.gob.cl/portal/url/item/89185 968d325a51ee04001011e010c92.pdf

15. Atalah E. Epidemiología de la obesidad en Chile. Rev Med Clin Condes 2012;23:117-123.

16. Contreras A, Herrera Y, Rodríguez L, Pizarro T, Atalah $\mathrm{E}$. Aceptabilidad y consumo de una bebida láctea con omega-3 en embarazadas y nodrizas del Programa Nacional de Alimentación Complementaria. Rev Chil Nutr 2011;38:313-320.

17. Atalah E, Vera G, Rosselot G, Araya H, Andreu R, Alviña M, Araya M, et al. Desarrollo, consumo y aceptabilidad de una bebida láctea con DHA para embarazadas y nodrizas. Rev Chil Nutr 2008;35:433-42. 